\title{
Traduire
}

Une eutre perspective sur $r$ tatadciction

Revue française de la traduction

$235 \mid 2016$

Luxe, mode... et traduction !

\section{La traduction des expressions figées : langue et culture}

Mohamed Saad Ali

\section{(2) OpenEdition}

1 Journals

Édition électronique

URL : https://journals.openedition.org/traduire/865

DOI : $10.4000 /$ traduire.865

ISSN : 2272-9992

Éditeur

Société française des traducteurs

Édition imprimée

Date de publication : 1 décembre 2016

Pagination : 103-123

ISSN : 0395-773X

\section{Référence électronique}

Mohamed Saad Ali, «La traduction des expressions figées : langue et culture », Traduire [En ligne],

235 | 2016, mis en ligne le 01 décembre 2018, consulté le 05 juillet 2021. URL : http://

journals.openedition.org/traduire/865 ; DOI : https://doi.org/10.4000/traduire.865 


\section{La traduction des expressions figées : langue et culture}

\section{Mohamed Saad Ali}

\section{Introduction}

Le lexique d'une langue ne se compose pas seulement de mots simples, mais aussi d'expressions et de mots composés. Autrement dit, il comprend aussi bien des unités monolexicales que des unités polylexicales. Le premier groupe est très largement abordé dans bon nombre d'études linguistiques diverses et variées. Quant au deuxième, il fut pendant longtemps laissé de côté, ou abordé partiellement dans quelques ouvrages spécifiques. Soulignons que cette " négligence " ne provient pas d'un manque d'importance, car le figement(1) est un procédé qui assure le renouvellement du lexique (Mejri, 2000 : p. 42), et " une langue qui ne produit plus de SF (Syntagmes Figés) est une langue vouée à la disparition " (Mejri, 2012 : p. 146).

Selon nos recherches, la théorie de la phraséologie aurait été élaborée pour la première fois par Bally dans son Traité de stylistique :

Si, dans un groupe de mots, chaque unité graphique perd une partie de sa signification individuelle ou n'en conserve aucune, si la combinaison de ces éléments se présente seule avec un sens bien net, on peut dire qu'il s'agit d'une locution composée. [...] C'est l'ensemble de ces faits que nous comprenons sous le terme de phraséologie. (1951, p. 65-66)

Donc, Bally parle d'une locution composée où la compositionnalité du sens est, partiellement ou totalement, absente. Alors que cette classification est basée sur l'aspect sémantique, il faut savoir que la notion de figement est forcément reliée à l'absence plus vaste d'une dynamique linguistique. Selon le dictionnaire de linguistique Larousse, "le figement est un processus linguistique qui, d'un syntagme dont les éléments sont libres, fait un syntagme dont les éléments ne peuvent être dissociés. Ainsi, les mots composés (compte rendu, pomme de terre, etc.) sont des syntagmes figés".

(1) Je tiens à remercier Gaston Gross, Professeur de linguistique à l'Université Paris-XIII, pour l'échange que nous avons eu sur le figement. 
On peut ainsi dire que les expressions dites figées refusent, en grande partie, la variation syntaxique et ont une signification opaque non déductible de la signification des unités lexicales qui les forment. Autrement dit, le figement rejette la manipulation syntaxique (le blocage de la combinatoire syntaxique) et se caractérise par une opacité sémantique (la non-compositionnalité du sens). Le figement jouit d'une position interdisciplinaire ; il concerne le lexique, la morphosyntaxe, la sémantique, mais aussi la pragmatique comme nous allons le voir un peu plus loin, là où le contexte de l'énonciation pourrait quelquefois déterminer le sens de ces expressions à terminologie flottante.

\section{Problématique}

La traduction des expressions figées pose des problèmes linguistiques et extralinguistiques. En analysant ces expressions, qu'elles soient en français ou en arabe, il est facile de déceler la solidarité entre la forme et le contenu, c'est ce qu'on appelle en linguistique la " fixité idiomatique " (Mejri : 2010). Cette dissociation des deux faces des signes linguistiques constitue une problématique majeure, notamment quand il s'agit de traduire entre deux langues appartenant à deux systèmes linguistiques très différents. De plus, beaucoup d'expressions figées sont chargées d'implicites sociaux et culturels. La situation se complique encore davantage lorsqu'il s'agit de transposer les expressions figées d'un texte religieux révélé il y a plus de 1400 ans. Nous sommes alors face à d'énormes différences linguistiques et culturelles.

Le but de notre étude est de dégager les problèmes que soulève la traduction d'expressions figées puisées dans le Coran en langue française. Pour ce faire, notre réflexion tâchera de répondre à quelques questions :

1 - Quelles sont les caractéristiques linguistiques de ces expressions?

2 - Est-ce que ces caractéristiques sont présentes dans les deux langues, arabe et française?

3 - Quelles sont les difficultés pour traduire ces expressions?

4 - Comment les traducteurs ont-ils transposé ces expressions figées coraniques?

Par ailleurs, il nous semble que l'importance de cette étude s'explique par la rareté des recherches portant sur la traduction d'expressions arabes figées en langue française, notamment celles provenant du Coran. Nous espérons ainsi que ce travail permettra d'atteindre deux objectifs : ouvrir la porte à d'autres études contrastives pour traduire de telles expressions du Coran, ce qui pourrait contribuer à l'élaboration d'un dictionnaire bilingue arabe-français des expressions figées coraniques, et faciliter la communication interculturelle. 


\section{Méthode d'analyse}

Dans un premier temps, nous procéderons à une étude théorique du figement à l'aide d'exemples tirés des langues française et arabe, pour pouvoir ensuite aborder les difficultés rencontrées lors de la traduction d'expressions figées. Dans un deuxième temps, nous analyserons quelques expressions coraniques et leur traduction française, en adoptant la méthode décrite ci-dessous. Soulignons que, pour des raisons évidentes, l'analyse se bornera à des exemples provenant d'un seul champ sémantique, à savoir les caractères moraux de l'Homme. Ces exemples sont puisés dans deux traductions françaises (des sens) du Coran : la première, que l'on doit à un traducteur français non-musulman : Jean Grosjean (2008), la deuxième, qui est l'œuvre d'un traducteur arabe musulman : Mohamed Chiadmi (2008). Ce choix ne résulte pas du hasard, car nous voulons voir à quel point la langue maternelle et la culture religieuse d'un traducteur peuvent influer sur le processus de traduction d'expressions structurées selon les règles propres de la langue concernée et enracinées dans la culture véhiculée par cette langue.

En nous appuyant sur la théorie interprétative où le sens est l'objet du processus de traduction et en admettant que la forme du texte de départ (les expressions figées) participe à la construction du sens, nous avons choisi une méthode d'analyse en quatre étapes :

1 - La translittération de l'expression figée

2 - La traduction mot-à-mot de l'expression figée pour transmettre la couleur locale

3 - La traduction interprétative : le sens global, le contenu culturel véhiculé et la situation d'énonciation de l'expression en question

4 - L'analyse et la comparaison des traductions proposées

\section{Les études sur le figement et sa traduction}

Depuis peu, la question du figement occupe une place de plus en plus importante dans les études linguistiques, en raison de sa richesse et de sa complexité. "Les expressions figées, habituellement écartées des discussions théoriques, constituent un domaine d'étude à part entière. " (Gross, 1982 : p. 184). D'ailleurs, il faut avouer que l'étude du figement en français dépasse de loin son étude en langue arabe, jusqu'à présent marginale, bien que le premier ouvrage sur ce thème ait été écrit en 1985 par Karim Hosam-el-Din sous le titre d'At-ta 'bir al-iștlahyy [L'expression idiomatique]. S'il a fallu attendre 1996 pour que Gaston Gross présente aux francophones son ouvrage complet Les expressions figées en français, noms composés et autres locutions, de nombreuses autres études se sont ensuite succédé dans les deux langues, avec une supériorité du nombre tout à fait remarquable en ce qui concerne la langue française. 
L'étude de Hosam el-Din (1985) est une réelle tentative visant à mettre en évidence et à analyser le phénomène du figement dans la langue arabe. II cherche à y définir la notion du figement, ses caractéristiques, ses champs sémantiques et ses différentes structures. Ghorâb (2005) essaie, pour sa part, d'aborder la structure et la sémantique des expressions figées dans le texte coranique.

Pour ce qui est de la langue française, les premiers linguistes ont commencé, bien entendu, par l'évocation des problèmes sémantiques et morphosyntaxiques du phénomène complexe du figement : sa classification, son flottement terminologique, ses caractéristiques, etc. Ensuite, d'autres linguistes et traductologues ont tenté de traiter ce phénomène par une approche comparative, comme l'ouvrage de Palma (2007) qui porte sur la comparaison entre le français et l'espagnol, ou bien par rapport à la possibilité de traduire ces expressions d'une langue vers une autre. Parmi les études concernant la traduction des expressions figées entre le français et l'arabe, nous nous permettrons d'en citer quelques exemples.

Le numéro 2 (vol. 53) de la revue META (2008) aborde globalement cette problématique. Parmi les articles mettant en parallèle le français et l'arabe, nous trouvons l'intervention de B. Ouerhani. Basée sur les problèmes linguistiques de la traduction automatique, elle présente une description des constructions à verbes support en arabe et en français. T. Ben Amor (2008), quant à elle, soulève le problème de l'équivalence des séquences figées dans la traduction intralinguale et interlinguale, en indiquant que la traduction des expressions figées est une manière de " dire autrement " soit au sein d'une même langue soit dans une autre langue. Au niveau de la traduction interlinguale, l'objet de notre recherche, elle montre, à partir d'exemples précis, que le figement peut correspondre à " deux modèles de construction de sens différents ", à " des unités lexicales de statuts différents " ou à " des choix stéréotypiques différents ".

Parmi les autres articles traitant de la problématique de la traduction des expressions figées, Mejri (2009) envisage le figement et le défigement du point de vue de la traduction. II essaie de détailler les mécanismes de chaque processus pour améliorer la description du figement, et par conséquent la traduction des séquences figées.

\section{Le flottement terminologique}

Vu l'importance et la complexité du figement, plusieurs termes sont disponibles pour dénommer le phénomène. En langue française, on parle d'" expressions figées " (G. Gross, 1996), de "séquences figées " (Mejri, 1997), d'" éléments figés " (Plama, 2007), etc. Pour ce qui est de l'arabe, il en va de même, les termes les plus répandus étant : لتركيب الدسكوك /At-tarkīb

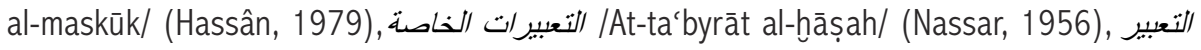
الاصططاحى/At-ta'bīr al-iștlāḥy/ (Hosam el-Din, 1985), etc. 
Force est de constater, en consultant les dictionnaires de linguistique, que chaque dénomination peut couvrir une des dimensions multiples du phénomène du figement. Mejri (1997), après avoir analysé un groupe de termes différents qui désignent le figement : locutions, expressions idiomatiques, idiotismes, mots composés, synapsie, synthème, lexie complexe, établit que les segments figés sont mal dénommés parce que chacun de ces termes ne couvre que quelques aspects du figement. Par voie de conséquence, il suggère le syntagme " séquence figée " qui, selon lui, pourrait répondre à toutes les dimensions du figement et engloberait presque toutes les autres dénominations.

De notre côté, selon la perspective à partir de laquelle nous approchons le figement, nous préservons l'adjectif " figé ", mais en y ajoutant le terme " expression ", parce que nous ne nous intéressons dans cette étude qu'aux types de formation qui n'ont pas perdu leur caractère polylexical et peuvent ainsi être qualifiés d'" expression ". II acquiert, de surcroît, le sens de " manière d'exprimer quelque chose " qui est plus vaste et peut ainsi répondre au caractère non compositionnel du figement. Quant à " séquence ", ce vocable convient mieux, nous semblet-il, si l'on parle d'un point de vue syntaxique, alors que notre étude n'est pas purement syntaxique. Soulignons aussi que nous excluons ici les proverbes, car ils sont considérés plus propres à la parémiologie (Palma, 2007).

Ainsi, le figement se caractérise par une terminologie flottante ; les linguistes eux-mêmes admettent la difficulté de déterminer clairement et de manière consensuelle ce phénomène. "Nous sommes nombreux à trouver que c'est un thème admirable, sans pouvoir dire avec netteté ce que c'est " (Martin, 1997 : p. 291). Le flottement terminologique n'est pas le seul aspect du figement, et dans ce qui suit, nous chercherons à identifier les caractéristiques principales des expressions figées.

\section{Les caractéristiques principales des expressions figées}

Il est intéressant, dès le début, de souligner que dans les études abordant la question du figement, on parle généralement d'expressions figées et de combinatoires libres (non figées). Cette dernière formule ne doit pas laisser penser qu'il s'agirait d'une liberté absolue dans l'organisation des unités lexicales sur l'axe syntagmatique(2). "Ce qu'on désigne souvent par combinatoire libre n'est en réalité qu'une combinatoire qui obéit à des contraintes d'ordre sémantique ou syntaxique " (Gross et Massoussi, 2011 : p. 96).

Cela dit, comment savoir si une expression est figée ou non ? Autrement dit, quels critères doivent être réunis pour dire d'une expression qu'elle est figée ? Pour pouvoir répondre

(2) Pour de plus amples considérations sur la relativité de ce terme, voir Salah Mejri (2011) : "Figement, collocation et combinatoire libre ", in Jean-Claude Anscombre, Salah Mejri (éd.), p. 63-77. 
systématiquement à cette question, regardons d'abord les éléments des deux paires d'expressions suivantes pour en déceler les nuances linguistiques:

$$
\begin{aligned}
& A \text { - Paul a cassé sa pipe } \quad B \text {-Paul a cassé la fenêtre } \\
& \text { أتى جميع الاصدقاء }
\end{aligned}
$$

En analysant ces énoncés, des différences nous paraissent évidentes sur deux axes :

1 - Axe sémantique : la manière de déduire le sens n'est pas identique dans les deux énoncés de chaque paire. Tandis que dans le premier élément de chaque paire, le sens global de l'expression n'est pas déduit de l'ensemble de sens de ses composants, dans le deuxième, le sens provient de ses unités lexicales.

2 - Axe morphosyntaxique : contrairement au premier élément de chaque paire, le deuxième jouit d'une entière liberté sur les deux axes, paradigmatique et syntagmatique.

Ces deux caractéristiques essentielles distinctives des expressions figées nous amènent à nous interroger sur les difficultés de la traduction de telles expressions et de la méthode la plus appropriée pour les transmettre d'une langue à l'autre.

\section{Figement et traduction}

\subsection{Difficultés de traduction des expressions figées}

En fonction des deux caractéristiques qui précèdent, nous pouvons résumer les difficultés à traduire les expressions figées de la manière suivante:

\subsubsection{L'opacité sémantique}

Les expressions figées se caractérisent par une globalité sémantique, c'est-à-dire que leur sens global ne provient pas du sens des unités lexicales qui la composent : elles sont donc noncompositionnelles. L'opacité sémantique d'une expression figée est ainsi bien rattachée à la non-compositionnalité de son sens (Tamba, 2011 : p. 116), qui recèle souvent un aspect

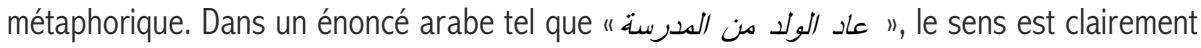
constitué par la combinaison du sens des mots le composant. Aussi une traduction littérale en langue française donnera-t-elle un sens pertinent : " L'enfant est rentré de l'école ". Mais dans un autre énoncé tel que : " ملحه علي ركبته " une interprétation littérale de la somme des mots ne donne pas le sens voulu, mais plutôt un sens inacceptable, voire étrange : "Son sel est sur son genou ". En effet, il s'agit d'une expression consacrée qui veut dire que la personne est سريع الغضب /sari'u al-ġaḍab/, soit facile à énerver, à mettre en colère. 
Il en va de même pour la langue française où une expression telle que " II a cassé sa pipe ", n'admet pas une lecture compositionnelle parce que l'on ne peut pas conclure le sens voulu à partir du sens de l'ensemble des mots ; tandis qu'une autre suite comme "Le garçon a offert le café ", peut être interprétée et comprise littéralement parce qu'il s'agit ici d'une combinatoire dite libre.

Il est intéressant de souligner que certaines expressions se prêtent à une double lecture : compositionnelle et non compositionnelle (figée) (Palma, 2007 : p. 23) ou, en d'autres termes, l'une transparente et l'autre opaque (G. Gross, 1996 : p. 11). Ce dernier donne comme exemple cet énoncé : "Les carottes sont cuites", qui peut avoir un sens compositionnel pour indiquer que ces légumes sont prêts à être mangés, et un autre sens opaque pour indiquer que la situation est désespérée.

\subsubsection{Les restrictions morphosyntaxiques}

L'étroite relation de l'opacité sémantique avec des restrictions morphosyntaxiques qui influent sur le verbe ou des arguments est aisément identifiable. En effet, l'expression figée n'accepte pas les transformations morphosyntaxiques qui concernent les phrases dites libres (passivation, pronominalisation, relativation, nombre du nom, temps du verbe, etc.). Prenons ici cet exemple : "Luc a pris la tangente ", (G. Gross, 1996 : p. 12-13), où nous pouvons observer l'absence de propriétés transformationnelles:

- La tangente a été prise par Luc.

- Qu'a pris Luc?

- Luc l'a prise.

- C'est la tangente que Luc a prise.

- La tangente que Luc a prise.

Soulignons aussi que le figement n'est pas absolu dans les expressions figées mais, comme le remarque G. Gross, les variantes sont plus fréquentes que le figement total (1996: p. 16). De plus, à partir de plusieurs exemples, Mogorrón Huerta (2011) aborde les variations possibles que peuvent subir les expressions figées : des variantes morphologiques (singulier/pluriel, masculin/féminin, etc.), des variantes morphosyntaxiques (déterminants, prépositions, variantes graphiques, etc.) et, dans une moindre mesure, des variantes lexicales. II en résulte que le figement n'a pas toujours le même degré.

Prenons aussi en considération la portée du figement. Moins fréquemment, nous trouvons que l'ensemble de l'expression est figé, comme dans cette locution verbale " avoir les yeux plus gros que le ventre ". Quelquefois le figement affecte seulement une partie de la phrase comme dans "être au bout du rouleau ". 


\subsubsection{La limitation paradigmatique}

Dans les phrases libres, les différents syntagmes peuvent facilement alterner avec d'autres qui appartiennent aux mêmes paradigmes. Par exemple, dans cet énoncé "L'enfant va à l'école ", nous pouvons opérer ces alternances paradigmatiques :

- Le garçon va à l'école.

- Les enfants vont à leur école.

- Le garçon part à l'école.

- Les filles vont au lycée.

Cette possibilité d'alternance paradigmatique est bien limitée dans la plupart d'expressions figées. L'expression " casser sa pipe " ne peut subir des variations comme *casser sa bouffarde ou *briser sa pipe. (G. Gross, 1996 : p.18)

De même, en langue arabe, une expression libre telle que : "يحب الطفل التفاح ", peut subir ces modifications sur l'axe paradigmatique :

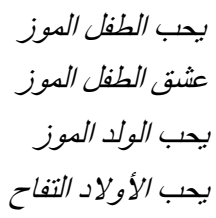

Mais, il en va tout autrement pour l'expression figée : " قضى نحبه ", " Respecter, effectuer son engagement ", qui veut dire " mourir " et qui n'accepte aucune alternance paradigmatique.

Soulignons aussi que ce critère de rupture paradigmatique n'a pas de valeur absolue parce qu'une certaine variation peut affecter le verbe, un des arguments, ou les deux à la fois (Lamiroy, Klein et al., 2010 : p. 16) : abîmer/arranger le portrait de quelqu'un, "battre quelqu'un "; s'occuper / se mêler de ses oignons "s'occuper de ses affaires".

De même, en langue arabe, l'expression figée suivante peut subir une variation au niveau du verbe sans affecter son sens : رجع/عاد بخفي حنين qui signifie "échouer ".

Après ce passage en revue des caractéristiques générales des expressions figées, examinons comment les transmettre d'une langue à l'autre :

\subsection{Comment traduire les expressions figées?}

Malgré la terminologie flottante qui caractérise le figement et la relativité qui frappe chaque élément de ses critères, nous sommes d'accord avec Tamba (2011: p. 109) sur l'existence d'un consensus concernant le double jeu de contraintes intervenant dans cette caractérisation 
"Les unes, internes, recensent des restrictions distributionnelles qui limitent les possibilités combinatoires tant grammaticales que lexicales. Les autres, externes, enregistrent des conventions culturelles et des routines communautaires ".

Donc, avant d'entamer la traduction des expressions figées, il faut bien maîtriser ces deux aspects interne (linguistique) et externe (culturel), c'est-à-dire assimiler la dimension sémantique du figement qui englobe tous les facteurs internes et externes. Cette dimension sémantique des expressions figées est assez complexe, mais elle est en même temps la plus importante au niveau de la traduction parce que, comme le dit le linguiste anglais J.R. Firth, " tout le problème de la traduction est d'ordre sémantique "(3) (Gregory : 1980).

D'ailleurs, si à la fin du siècle dernier, Mejri juge que l'absence de travaux exhaustifs sur le figement provient de la complexité de ce phénomène (1997 : p. 9), l'étude de la traduction des expressions figées n'est pas facilitée par le fait qu'elle remet en question à la fois la problématique du figement et celle de la traduction avec ses difficultés linguistiques et extralinguistiques. En abordant la question de la traduction des expressions figées, nous nous trouvons donc en face d'une double interdisciplinarité. La première, interne, concerne le figement en tant que phénomène linguistique complexe embrassant tout le système linguistique d'une langue quelconque (Mejri, 2008 : p. 244) et où la formation des expressions figées est soumise à des mécanismes linguistiques ayant trait au lexique, à la syntaxe, à la morphologie, à la sémantique et aussi à la phonologie. L'autre interdisciplinarité, externe, concerne cet aspect du processus de traduction qui fait appel à des disciplines autres que la linguistique : la sociologie, la psychologie, l'ethnologie, l'histoire de la culture, etc. C'est la raison pour laquelle les travaux exhaustifs sur la traduction des expressions figées sont rares, contrairement aux études portant sur la traduction des unités monolexicales.

Par ailleurs, la difficulté de traduire les expressions figées ne réside pas seulement dans le fait de transcoder isolément les unités lexicales d'une langue dans une autre. La traduction de ces expressions suit la même démarche que toute traduction humaine impliquant la mise en œuvre d'un " processus cognitif " qui " consiste pour le traducteur à comprendre un texte/discours pour le faire comprendre à des destinataires qui n'ont pas accès à l'original " (Durieux, 2008 : p. 324).

Par conséquent, nous pouvons diviser l'opération traduisante des expressions figées en deux étapes principales :

La première étape est celle de la compréhension de l'expression. Celle-ci s'articule autour des deux aspects soulignés plus haut, à savoir les aspects linguistique et culturel.

(3) Cité par Michael J. Gregory, "Perspectives on Translation from the Firthian Tradition ", in META, 4(25), 1980, p. 455. Texte original: "The whole problem of translation is in the field of semantics". 
Pour l'aspect linguistique, la compréhension touche en premier lieu à la syntaxe de la langue de départ. II faut que le traducteur isole la séquence figée et la traite comme un ensemble indissociable. Par exemple, si le traducteur ne reconnaît pas le figement de l'expression " casser sa pipe ", il arrivera bien entendu à un faux-sens ou contresens. La traduction des unités lexicales composant cette expression nous donne une séquence n'ayant aucune relation avec son sens global.

Quant à l'aspect culturel, il va sans dire que la traduction répond généralement à des besoins culturels, politiques, scientifiques, etc. De même, la méthode de traduction peut différer d'un domaine à l'autre. Pour réaliser une communication efficace, le traducteur doit, dans un premier temps, bien analyser et comprendre le contenu culturel sous-tendant telle ou telle expression figée.

Une fois cette étape franchie, on peut passer à la suivante qui consiste en la réexpression de ce que l'on a compris. Le traducteur réexprime ainsi le contenu culturel d'une expression figée selon les mécanismes discursifs acceptés par la culture réceptrice. II faut par conséquent maîtriser ce qu'on appelle le " transfert culturel " (Vaguer, 2011 : p. 391).

Pour mettre en pratique le processus ainsi décrit, nous pouvons nous appuyer sur la traduction de l'expression figée arabe suivante en français : له عنق فى الخير/lahu 'unuqun fy alhaîr/. En traduisant mot à mot cette expression, on obtient la séquence : " l| a un cou dans le bien ", qui n'évoque rien chez le lecteur français. Mais si l'on revient à l'analyse du contenu culturel, nous constaterons que les arabophones utilisent cette expression pour désigner les personnes qui se montrent généreuses et charitables envers les autres, ce qui correspondrait en langue française à " avoir le cœur sur la main ". C'est ce qu'on appelle "l'équivalence " dans la théorie interprétative de la traduction.

\section{3. À la recherche des équivalences}

Comme chacun le sait, la traduction interlinguale pose des problèmes du simple fait de la présence de deux systèmes linguistiques différents. Pour la traduction des expressions figées, la situation est encore plus compliquée et moins évidente, notamment entre des langues n'ayant pas le même patrimoine historico-culturel. La traduction des expressions figées de l'arabe vers le français dépasse ainsi les problèmes dus aux différences de catégorisation et de grammaticalisation entre ces langues, le figement étant un " mécanisme cristallisant l'idiomaticité d'une langue " (Mejri, 2008 : p. 245).

En abordant la traduction de telles expressions, la première solution qui viendra à l'esprit d'un traducteur sera de rechercher des équivalences. Cette méthode de traduction, mise en exergue depuis la Stylistique comparée, est à nos yeux plus efficace parce qu'elle peut, en grande partie, réduire les pertes sémantiques des unités traduites. II est intéressant, au passage, 
d'établir la distinction entre les équivalences et les correspondances, à la base de la théorie interprétative de la traduction. Les équivalences sont des faits de discours qui proviennent d'une créativité d'expression, tandis que les correspondances sont des faits de langue, comme c'est le cas des vocables pris isolément hors discours(4). Mais, est-ce que cette stratégie est toujours et facilement réalisable? En prenant l'exemple des équivalences entre des formes sentencieuses appartenant au français et à l'espagnol, deux langues partageant pourtant un fonds commun latin et la même culture classique gréco-latine, Anscombre (2008) conclut à des limites soit au plan linguistique, soit au plan stylistique et dénombre aussi des cas épineux. Par conséquent, s'agissant de chercher des équivalences entre l'arabe et le français, deux langues ne partageant ni le même fonds commun ni la même culture, les limites linguistiques et stylistiques risquent d'être ipso facto plus marquées et les cas épineux beaucoup plus nombreux.

En outre, il convient de constater que si le critère de l'opacité sémantique des expressions figées peut varier au sein d'une même langue en fonction de la variation géographique (Lamiroy, Klein et al., 2010 : p. 14), la situation est plus délicate si l'on y ajoute également une variation socio-historique et culturelle, comme dans le cas de la traduction des expressions figées arabes citées au sein du texte coranique.

\subsection{Le figement dans le Coran}

Pour ce qui concerne le figement dans le texte coranique, il nous semble indispensable de mettre l'accent sur la portée de ce phénomène dans le texte en question. Nous observons qu'il est particulièrement rare d'y trouver des expressions entièrement figées, parce que le Coran ne cite pas de dictons ou de maximes hors contexte. Pour assurer une cohérence textuelle, chaque sourate (chapitre coranique) constitue une unité énonciative(5) où l'on peut trouver des histoires des prophètes précédents, des récits vécus, des événements ultérieurs, ou bien des lois divines réglementant la vie des croyants. Donc, si le texte se sert des expressions figées, il les intègre dans un de ces contextes ; ces expressions sont ainsi manipulées dans le Coran de manière à répondre aux besoins communicatifs et argumentatifs de la langue coranique. Dans la partie qui suit, nous essayerons d'analyser quelques expressions figées coraniques et les traductions retenues.

(4) Pour de plus amples détails sur la distinction entre équivalences et correspondances dans la théorie interprétative de la traduction, voir Marianne Lederer, 2002.

(5) Pour de plus amples détails sur le phénomène de la cohérence textuelle dans le Coran, voir As-Syūty, 1974, 3/369-389. 
$1-$

\begin{tabular}{|l|l|}
\hline خفض جَنَاحَه & ḩafaḍa janaḥahu \\
\hline
\end{tabular}

\section{Le segment de verset :}

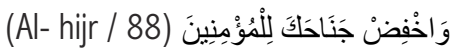

Traduction mot-à-mot : abaisse ton aile

Traduction interprétative : Dans ce segment de verset, Allah demande au Prophète, à travers une expression arabe ancienne, d'avoir bon cœur à l'égard des croyants. Selon les exégètes, il empêche le Prophète d'être arrogant et l'incite à la modestie. Cette expression a un caractère métaphorique car le Prophète est désigné comme un oiseau et les croyants comme ses enfants qu'il recouvre de son aile comme dans un geste d'amour et de pitié.

\section{Les traductions :}

Chiadmi : sois bienveillant à l'égard des croyants

Grosjean : veille seulement sur les croyants

II est clair que les deux traducteurs n'ont pas eu recours aux équivalences qui auraient mieux permis de conserver le sémantisme des expressions figées et auraient donné de bons résultats en termes d'adéquation entre les systèmes lexicaux (Mejri, 2008 : p. 249). À la place, ils utilisent, chacun à leur manière, une traduction interprétative pour rendre le texte de départ dans une langue française accessible et acceptable. D'ailleurs, on ne retrouve pas l'effet provoqué par le texte de départ dans la traduction en raison de la perte du caractère métaphorique. Soulignons aussi que, dans la version de Grosjean, l'utilisation de l'adverbe seulement charge la traduction d'une portée comparative absente du verset initial.

$2-$

\begin{tabular}{|l|l|}
\hline لان جلده & lana jildahu \\
\hline
\end{tabular}

\section{Le segment de verset :}

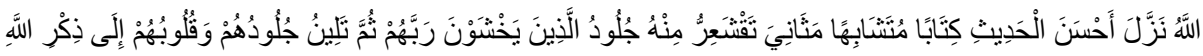
(Les groupes / 23)

Traduction mot-à-mot : leurs peaux s'adoucissent

Traduction interprétative : Selon les exégètes, cette expression veut dire que pour les croyants dont parle le verset, la lecture des belles nouvelles citées dans le Coran transformera leur 
angoisse et leur peur en tranquillité et en espoir. L'expression est ainsi basée sur la relation éventuelle des émotions humaines avec la peau. Consultons les traductions proposées:

\section{Les traductions :}

Chiadmi : puis leurs peaux et leurs cœurs s'apaisent à l'évocation

Grosjean : mais leur peau et leur cœur s'attendrissent à l'avertissement de Dieu

Avec l'utilisation du verbe "s'apaiser ", Chiadmi a bien compris et rendu le sens voulu dans le texte source. Quant à Grosjean, il nous semble que sa version n'est pas suffisamment pertinente car même si le verbe "attendrir " à la forme pronominale peut bien signifier " toucher " ou " émouvoir ", on trouve également des expressions comme "II s'attendrit sur son sort " où le sens est plus proche du verbe "s'apitoyer ", ce qui donne une connotation négative. Or, le verbe utilisé dans l'expression initiale لان / lana/ montre bien l'effet positif de l'évocation d'Allah qui conduit à la tranquillité et au calme psychologique.

$3-$

اعتصم بحبل الله

i‘tașama bihabli ellah

\section{Le segment de verset :}

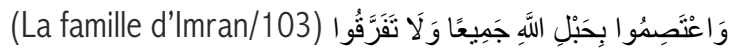

Traduction mot-à-mot : il tient la corde d'Allah

Traduction interprétative : Ce segment de verset est un ordre divin aux croyants leur sommant de conclure un pacte avec Allah, c'est-à-dire de croire en Lui et de Lui obéir. Cette expression a un sens métaphorique basé sur un élément de l'environnement arabe, الحبل/habl/ "la corde ". Cet aspect métaphorique provient du fait qu'une corde et un pacte sont tous deux des outils pour relier, soit concrètement soit abstraitement, des choses ou des personnes. Les lexicologues, pour leur part, estiment que cette expression signifie qu'il faut laisser de côté la dispersion et suivre les instructions du Coran (Ibn Manzour : 11/135).

\section{Les traductions :}

Chiadmi : Attachez-vous tous fermement au pacte de Dieu.

Grosjean : Mettez-vous tous sous la protection de Dieu.

Aucun des deux traducteurs ne donne une équivalence capable de conserver l'effet recherché dans le texte de départ. Par ailleurs, Chiadmi propose une traduction interprétative, mais avec l'utilisation du verbe "s'attacher ", il nous semble qu'il n'a pas pris en considération l'évolution de sens du concret vers l'abstrait. II aurait dû utiliser, à titre d'exemple, le verbe " réaliser " ou " conclure ". Quant à Grosjean, sa version contient clairement un faux-sens, il a mal compris la 
dimension culturelle de l'expression originale. Pourquoi ne dirait-on pas : "Réalisez tous ensemble le pacte de Dieu et ne vous en détournez pas ". II est, toutefois, frappant de souligner qu'il y a toujours une perte au niveau de la couleur locale.

$4-$

\begin{tabular}{|l|l|}
\hline ختم على قلبه & hatama 'ala qalbih \\
\hline
\end{tabular}

Le verset :

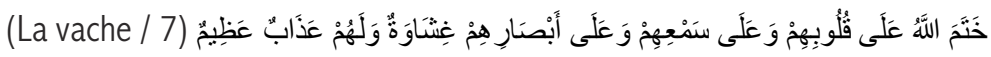
Traduction mot-à-mot : sceller son cœur

Traduction interprétative : Selon les lexicologues, cette expression désigne celui qui ne comprend plus, comme si son esprit était scellé ou bloqué (Ibn Manzour : 163/12). II est intéressant de souligner que le Coran se sert du terme قلب /qalb/, " cœur ", pour désigner l'esprit. Dans le contexte de ce verset, cette ancienne expression métaphorique signale l'absence de raisonnement et de compréhension des non-croyants qui s'éloignent du droit chemin.

\section{Les traductions :}

Chiadmi : et Dieu a scellé leur cœur

Grosjean : Dieu a scellé leur cœur

Les deux traducteurs ont recours ainsi à la littéralité sans présenter aucune note explicative. En effet, si la traduction littérale peut être considérée comme une solution possible, elle risque de choquer les récepteurs du texte d'arrivée. Ils ont ainsi tourné le dos à la non-compositionnalité de cette expression figée parce que les deux expressions originale et traduite ne sont pas équivalentes et ne suscitent donc pas le même effet que l'original auprès des lecteurs. Si les dictionnaires n'offrent pas d'équivalent pour l'expression en question, on peut recourir à des collocations permises en langue française, tout en prenant en considération les informations contextuelles ; on pourrait dire, par exemple, "Allah les prive définitivement de jugement ", ou bien "Allah scelle leur esprit ".

$5-$

يده مغلولة إلى عنقه

yadahu maǵlulatun 'la ‘unuqihi

Le segment de verset :

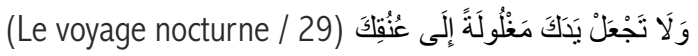

Traduction mot-à-mot : sa main est liée à son cou 
Traduction interprétative : Cette suite de mots à caractère métaphorique désigne l'avarice. Selon les lexicologues, la main serait maintenue vigoureusement autour du cou de manière à éviter de s'en servir pour engager des dépenses (Ibn Manzour : 11/504). C'est pourquoi cette expression est mise à l'impératif négatif pour demander aux musulmans de bannir ce trait de caractère défavorable.

\section{Les traductions :}

Chiadmi : Ne tiens pas la main collée à ton cou par avarice.

Grosjean : Ne garde pas ta main fermée vers ton cou.

II est clair que les deux traducteurs se servent de la solution de la littéralité, mais Chiadmi ajoute une information complémentaire par avarice en guise d'explication du caractère métaphorique de l'expression initiale, et ce dans le corps de la traduction et non pas dans une note. Le lecteur comprendrait facilement que cette partie ajoutée de la part du traducteur fasse partie du verset initial. II nous semble qu'au lieu de la mention par avarice, l'utilisation d'une expression idiomatique sur l'avarice comme "être près de ses sous " serait une solution plus adaptée. En effet, la langue française ne dispose pas d'une telle expression reliant l'avarice ou la générosité à la main. Une note explicative est, nous semble-t-il, indispensable pour faire comprendre la dimension extralinguistique de l'expression originale.

$6-$

\begin{tabular}{|l|l|}
\hline صعر خدa 'ara hadahu \\
\hline
\end{tabular}

\section{Le segment de verset :}

\section{وَلَا تُصَعِبِزْ خَدَّكَ لِلنَّاس (Luqman /}

Traduction mot-à-mot : faire dévier, détourner sa joue

Traduction interprétative : Les Arabes utilisaient cette expression pour souligner un trait de caractère négatif, à savoir l'orgueil. Elle symbolise le fait de détourner le visage des autres en signe d'orgueil (Ibn Manzour : 4/456). Ainsi le texte coranique demande-t-il aux musulmans de ne pas commettre cette erreur morale, et, par contre, de regarder les personnes auxquelles on s'adresse en signe de modestie.

\section{Les traductions :}

Chiadmi : Ne prends pas un air arrogant en abordant tes semblables!

Grosjean : Ne détourne pas ton visage des hommes.

Les deux traducteurs prennent des décisions opposées : Chiadmi utilise une traduction interprétative, tandis que Grosjean a recours à la littéralité. Le premier traducteur préserve le sens 
mais perd la couleur locale, l'autre garde la couleur locale mais perd le sens. En effet, ce qui compte dans le processus de traduction, c'est la transmission du sens, il ne faut pas sacrifier celui-ci pour garder la forme du texte de départ.

$7-$

\begin{tabular}{|l|l|}
\hline غليظ القلب & galîz̧alqalb \\
\hline
\end{tabular}

\section{Le segment de verset :}

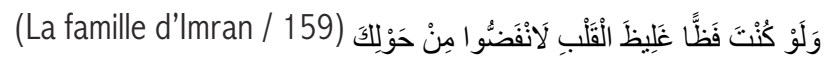

Traduction mot-à-mot : avoir un cœur dur

Traduction interprétative : Ce segment de verset met en exergue l'importance de la douceur dans le traitement des autres. Allah confirme à son Prophète que s'il avait un cœur de pierre, les gens ne le suivraient jamais. Notons, au passage, que le cœur est le siège de l'affectivité (sentiments, passions) dans plusieurs expressions, autant en arabe qu'en français. Cette formule sert donc à exprimer la dureté et la grossièreté envers les autres.

\section{Les traductions :}

Chiadmi : Car si tu te montrais brutal ou inhumain avec eux, ils se seraient tous détachés de toi.

Grosjean : Si tu t'étais montré rude et dur, ils se seraient séparés de toi.

Ici les deux traducteurs sont soucieux de transmettre le sens recherché aux récepteurs puisqu'ils ont recours à la traduction interprétative, mais la langue française dispose d'expressions contenant le substantif " cœur ", qui pourraient constituer des équivalents à l'expression arabe, comme " avoir le cœur dur, un cœur de pierre ".

$8-$

\begin{tabular}{|l|l|}
\hline عض على يديه & 'aḍa 'ala yadayhi \\
\hline
\end{tabular}

Le verset :

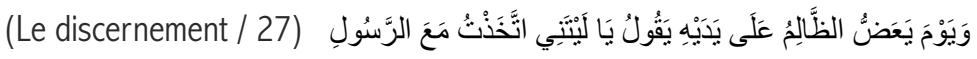

Traduction mot-à-mot : se mordre les deux mains

Traduction interprétative : Les êtres humains accompagnent quelquefois leur parole exprimant l'affectivité d'un geste du corps. Les exégètes expliquent que cette expression a une portée psychologique et possède aussi un caractère métaphorique indiquant le repentir, la tristesse 
et le regret de ce qui a été perdu. Dans le contexte de ce verset, les non-croyants sont malheureux et pleins de regrets de n'avoir pas suivi le Prophète.

\section{Les traductions :}

Chiadmi : C'est le jour où l'injuste se mordra les doigts en s'écriant : (...)

Grosjean : Ce jour-là, le coupable se mordra les mains.

Chiadmi et Grosjean utilisent respectivement " se mordre les doigts " et " se mordre les mains". En analysant ces deux choix, nous trouvons que le premier existe bien dans la langue française, mais dans un registre familier, tandis que l'autre n'est qu'une traduction littérale de la part du traducteur. En tout état de cause, les deux traductions peuvent se comprendre grâce au contexte du verset.

\section{فؤاده فارغ}

fu'aduhu fârig

\section{Le segment de verset :}

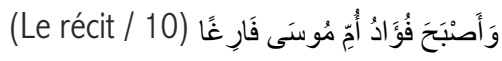

Traduction mot-à-mot : son cœur est vide

Traduction interprétative : Selon les lexicologues, cette expression souligne l'impatience (Ibn Manzour : 8/445). Ce segment de verset raconte la réaction de la mère du Prophète Moïse lorsqu'elle apprend que son bébé est tombé aux mains du Pharaon qui pourrait le tuer comme les autres enfants. Très inquiète, elle se sent incapable de patienter pour récupérer son bébé.

\section{Les traductions :}

Chiadmi : Pendant ce temps, le cœur de la mère de Moïse était accablé de douleur.

Grosjean : La mère de Moïse eut le cœur vide.

Nous nous trouvons ainsi devant deux situations différentes : l'interprétation de Chiadmi et la littéralité de Grosjean. Si Chiadmi a bien fait comprendre aux lecteurs le vouloir-dire du texte source, la version de Grosjean nous fait comprendre que la mère de Moïse serait dépourvue de toute émotion : c'est donc un contresens. D'autre part, les deux traducteurs ne donnent pas d'équivalent préexistant en langue française, leurs deux traductions sont des combinatoires libres. Pourquoi ne dirait-on pas " la mère de Moïse avait le cœur gros ou le cœur lourd (à cause du chagrin) "? 


\section{Conclusion}

Nous avons essayé dans notre étude d'effectuer un parcours comparatif, avec des exemples français et arabes, du phénomène de figement, qui retient depuis peu l'intérêt des linguistes.

Par conséquent, en analysant la traduction de ces expressions, nous nous appuyons en premier lieu sur l'aspect sémantique, nœud gordien du processus de traduction, et considérons alors la structure en étroite relation avec le sens. Vu le fonctionnement linguistique des expressions figées, nous en déduisons sans difficulté que la traduction de telles expressions soulève des problèmes formels. D'ailleurs, il nous semble que les expressions figées font partie des " mécanismes qui n'ont pas la même forme d'une langue à l'autre mais qui pourraient être transposés d'une langue à l'autre au sens où les effets de mécanismes réellement équivalents sont les mêmes d'une langue à l'autre " (Ibrahim, 1999 : p. 759). Ainsi, si la différence formelle des deux systèmes linguistiques arabe et français pose des problèmes spécifiques dans la traduction des expressions figées, cela n'empêche pas leur transfert grâce à des équivalences ayant le même effet que les expressions initiales.

D'ailleurs, nous observons généralement que la traduction de Chiadmi, dont la langue maternelle est l'arabe, est plus efficace que celle de Grosjean qui est francophone. Le recours de ce dernier à la littéralité sans note explicative dépasse-t-il de beaucoup celui de Chiadmi ?

D'après notre analyse de la traduction de quelques expressions figées, il nous semble, d'une part, que les deux traducteurs ne suivent pas toujours la même méthodologie : tantôt ils se servent de la littéralité, tantôt ils essaient de chercher des équivalences. D'autre part, nous pensons que le recours à l'équivalence est la première solution à laquelle le traducteur devrait penser. Mais les deux traducteurs ne trouvent pas toujours d'équivalence pertinente. De façon générale, aucun des deux ne réussit à transmettre efficacement aux destinataires tant la charge sémantique que l'effet du texte de départ à ses lecteurs.

Afin que le traducteur puisse choisir des équivalences pertinentes, il lui faut bien maîtriser les usages des deux langues impliquées dans le processus de traduction, et naturellement les cultures source et cible, parce que " dans différentes langues on n'utilise pas les mêmes significations pour exprimer les mêmes idées" (Seleskovitch, 2014 : p. 133).

II nous reste à ajouter que la traduction des expressions figées figurant dans un texte sacré, tel que le Coran, doit se faire avec plus de précision et de fidélité, notamment si l'on s'adresse à un public croyant ou néophyte. Pour traduire de telles expressions dans le texte coranique, nous proposons une méthode en trois étapes, dont les deux premières pourraient s'appliquer à n'importe quel processus traductionnel. La démarche sera donc la suivante :

1 - Une compréhension parfaite du sémantisme et de la structure morphosyntaxique de l'expression figée. Une connaissance suffisante de la culture arabe - celle de la langue de 
départ - est indispensable pour comprendre la portée culturelle d'une expression, et par la suite, le véritable message du texte de départ.

2 - Une fois l'expression bien comprise, le traducteur peut passer à la deuxième étape, à savoir la recherche d'une équivalence pertinente dans la langue française, (langue d'arrivée), équivalence qui doit avoir, autant que possible, le même effet que l'expression initiale. Ceci exige que le traducteur ait une connaissance suffisante de la langue et de la culture cibles afin de pouvoir communiquer ce qu'il a compris.

3 - Si la langue réceptrice ne dispose pas d'équivalence directe, le traducteur a deux choix : soit traduire littéralement l'expression figée pour transmettre la couleur locale aux destinataires, tout en accompagnant cette traduction d'une phrase explicative pour expliciter le vouloir-dire du texte source, soit l'inverse, c'est-à-dire inclure la version explicative au texte et, dans une note, proposer une traduction littérale. Par ce biais, il nous semble, d'une part, que le traducteur sera plus fidèle au texte source et, d'autre part, que le lecteur recevra le maximum d'informations contenues dans ce texte sacré et pourra ainsi partager en grande partie l'effet ressenti par les destinataires du texte coranique original.

hma_saad@yahoo.com

Mohamed Saad ALI est Maître de conférences à la faculté des Lettres, université du Fayoum en Égypte. Spécialité : Linguistique et traductologie.

\section{Bibliographie}

\section{Textes de référence}

GROSJEAN Jean, (trad.), 2008, Le Coran, Paris, Gallimard, Collection Folio.

CHIADMI Mohamed, (trad.), 2008, Le Noble Coran, Nouvelle traduction du sens de ses versets, Lyon (France), Éditions Tawhid.

\section{Références en français et en anglais}

ANSCOMBRE Jean-Claude, 2008, "Les formes sentencieuses : peut-on traduire la sagesse populaire ? ", in Meta, 53, p. 253-268.

BALLY Charles, 1951, Traité de stylistique française, Paris, Klincksieck.

BEN AMOR Thouraya, 2008, " Défigement et traduction intralinguale et interlinguale ", in Meta, 53, p. 443-455. 
DUBOIS Jean, 2002, Dictionnaire de linguistique, Paris, Larousse.

DURIEUX Christine, 2008, "Mettre la main sur le figement lexical : la démarche du traducteur ", in Meta, 53, p. 324-332.

GRAUSSE Maurice, 1982, "Une classification des phrases figées du français ", in Revue québécoise de linguistique, 11, p. 151-185.

GROSS Gaston, 1996, Les expressions figées en français, noms composés et autres locutions, Paris, Ophrys.

GROSS Gaston, MASSOUSSI Taoufik, 2001, "Figement et transparence " in ANSCOMBRE JeanClaude, MEJRI Salah, (éd.), Le figement linguistique : La parole entravée, Paris, Éditions Champion, p. 95-108.

IBRAHIM Amr Helmy, 2001, "Quelle linguistique pour la traduction ? ", in CABANILLAS Isabel de la Cruz, GARCIA Carmen Santamaria et al., (éd), La linguistica aplicada a finales del siglo XX. Ensayos y propuestas, Tome 2, Asociación Española de Lingüística Aplicada, p. 759-771.

LAMIROY Béatrice, KLEIN Jean-René et al, 2010, Les expressions verbales figées de la francophonie, Belgique, France, Québec et Suisse, Paris, Ophrys.

LEDERER Marianne, 2002, "Correspondances et équivalences : Faits de langue et faits de discours en traduction ", in ISRAËL Fortunato, (éd.), Identité, altérité, équivalence ? La traduction comme relation : actes du colloque internationale tenu à l'ÉSIT, les 24, 25 et 26 mai 2000 en hommage à Marianne LEDERER, Paris-Caen, Lettres modernes Minard, p. 17-28.

MARTIN R., 1997, "Sur les facteurs du figement lexical ", in MARTIN-BALTAR M., (éd.), La locution entre langue et usagers, Paris, ENS éditions, p. 291-305.

MEJRI Salah, 2011, " Figement, collocation et combinatoire libre ", in ANSCOMBRE Jean-Claude, MEJRI Salah, (éd.), Le figement linguistique : La parole entravée, Paris, Éditions Champion, p. $163-77$.

MEJRI Salah, 1997, Le figement lexical : descriptions linguistiques et structuration sémantique, Tunis, Publications de la Faculté des lettres de Manouba.

MEJRI Salah, 2000, "Figement et renouvellement du lexique : quand le processus détermine la dynamique du système ", in Le français moderne, 68, p. 41-62.

MEJRI Salah, 2008, "Figement et traduction : problématique générale », in Meta, 53, p. 244-252. GREGORY Michael J., 1980, "Perspectives on Translation from the Firthian Tradition », in Meta, 25, p. 455-466.

MOGORRÓN HUERTA Pedro, 2011, "Les expressions figées le sont-elles vraiment ? ", in ANSCOMBRE Jean-Claude, MEJRI Salah, (éd.), Le figement linguistique : La parole entravée, Paris, Éditions Champion, p. 218-234. 
OUERHANI Béchir, 2008, "Les problèmes linguistiques de la traduction automatique des prédicats nominaux entre l'arabe et le français ", in Meta, 53, p. 407-419.

PALMA Silvia, 2007, Les éléments figés de la langue, étude comparative français-espagnol, Paris, L'Harmattan.

MEJRI Salah, 2005, "Figement, néologie et renouvellement du lexique », in Linx, 52, p. 163174.

SELESKOVITCH Danica, 2014, "Interpréter un discours n'est pas traduire une langue ", in SELESKOVITCH Danica, LEDERER Marianne, (éd.), Interpréter pour traduire, Paris, Les belles lettres.

TAMBA Irène, 2011, "Sens figé : idiomes et proverbes ", in ANSCOMBRE Jean-Claude, MEJRI Salah, (éd.), Le figement linguistique : La parole entravée, Paris, Éditions Champion, p. 109-126.

VAGUER Céline, 2011, "Expressions figées et traduction : langue, culture, traduction automatique, apprentissage, lexique ", in ANSCOMBRE Jean-Claude, MEJRI Salah, (éd.), Le figement linguistique : La parole entravée, Paris, Éditions Champion, p. 391-411.

\section{Références en arabe}

AS-SYUTY Galal Ad-Dîn, 1974, Al- 'itqān fy 'ulūm al-Qur'ān [La perfection dans les sciences du Coran], Le Caire, Al-hayaa al-masryia lelkitâb.

GHORÂB Azza, 2005, At-ta 'bìrāt al-ișțtāthiyah fy Al-Qur'ān [Les expressions idiomatiques dans le Coran], Damiette (Égypte), La librairie de Nancy.

HASSAN Tammam, 1979, Allugatu Al-'arabyat :ma'naha wa mabnaha [La langue arabe : sa sémantique et sa syntaxe], Le Caire, Al-hayaa almasrya.

HOSAM EL-DIN Karim, 1985, At-ta ‘bìr al-ișțāhy [L'expression idiomatique], Le Caire, la librairie d'Al-Anglo al-masrya.

NASSAR Hussein, 1956, Al-mu 'jam al- 'araby: naš'atuhu watațawruhu [Le dictionnaire arabe : sa création et son développement], Le Caire, Dar Misr lelțiba‘ah. 